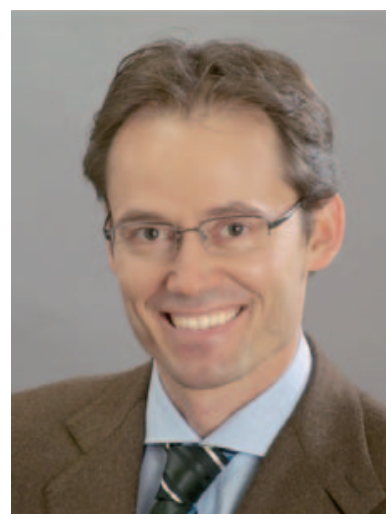

Dr. med. Marc Schlaeppi, St. Gallen, Schweiz

\section{Entwicklung von integrativer Medizin bedeutet auch Verantwortung für Qualität}

Die positive Entwicklung der komplementären und integrativen Medizin in den letzten zwei Jahrzehnten ist nicht mehr zu übersehen. Studien haben ermöglicht, die externe Evidenz zu vergrössern, und es wurden beispielweise für die integrative Onkologie Richtlinien und Empfehlungen in hochrangigen Journals publiziert. Wer heute immer noch behauptet, dass es keinen einzigen wissenschaftlichen Beleg zur Wirksamkeit in der Komplementärmedizin gibt, outet sich als unbelesener Ignorant auf dem Feld, das er kritisiert. Die Haltung hat sich also tendenziell sowohl auf konventioneller als auch auf komplementärer Seite gewandelt. Es soll nicht mehr gegeneinander, sondern synergistisch miteinander zugunsten des Patienten gearbeitet werden.

Selbstverständlich ist, dass jeder Mensch das Recht auf eine moderne konventionelle «state of the art»-Medizin hat - diese stellt die diagnostische und therapeutische Grundlage dar. Der andere, salutogene und ergänzende Blick des Komplementärmediziners trägt dazu bei, die Selbstheilungskräfte des Individuums zu fördern und zu stärken. Hier gibt es eine Vielfalt an erprobten Therapien und Methoden aus einer multimodalen integrativen Medizin. Diese Erweiterung ist nicht hoch genug einzuschätzen, denn sie ermöglicht häufig dem Patienten, wieder «Herr oder Frau im eigenen Haus» zu werden. Dort, wo es droht, kalt und sinnlos zu werden, wo die Angst beginnt, alles zu übertönen, kann eine differenzierte integrative Medizin wirksam und nicht selten Zuversicht und Hoffnung erzeugend eingesetzt werden. Dies kann wieder Schicksalsmut geben und ist eine Wohltat für unsere Patienten - und nicht zuletzt ein Beitrag zur Vermenschlichung der Medizin.

In den USA führt mittlerweile jedes Comprehensive Cancer Center eine Abteilung für integrative Medizin, in welcher Komplementärmedizin angeboten und beforscht wird. In der Schweiz und in Deutschland werden Lehrstühle und Professuren geführt, die ihren Auftrag für Lehre, Forschung und Patientenbetreuung wahrnehmen. Auch gibt es immer mehr öffentliche Abteilungen und Spitäler, die ein Angebot für integrative Medizin ermöglichen. Dies ist alles erfreulich, bedeutet aber auch Verantwortung für Qualität und Wissenschaft. Die Qualität für die ärztliche Weiter- und Fortbildung in Komplementärmedizin wurde in der Schweiz vorbildlich zwischen der Union schweizerischer komplementärmedizinischer Ärzteorganisationen (UNION) und dem Berufsverband der Schweizer Ärzteschaft (FMH) gelöst. Für einen Fähigkeitsausweis ist ein konventioneller Facharzt oder Äquivalent Voraussetzung, sodass die Träger eines Fähigkeitsausweises für Komplementärmedizin sich obligatorisch integrativ weitergebildet haben. Ein wichtiger Aspekt, insbesondere in der Onkologie, ist derjenige der Sicherheit. Fragen zu Neben- und Wechselwirkungen sind häufig aufwendig und schwierig zu beantworten, da immer wieder die klinischen Daten dazu fehlen. Hier besteht ein klarer Bedarf an weiterer Forschung und differenzierter Orientierung. So erfreulich die einen positiven Studienergebnisse zur Komplementärmedizin sind, müssen auch negative Resultate ernst genommen und schonungslos diskutiert werden - mit Konsequenzen. Integrative Spitäler, Einheiten und Abteilungen sind lernende Organisationen, in denen täglich Fragen, Hypothesen und mögliche Antworten während der Patientenbetreuung entstehen. Bekanntlich wird der Keim wahrer klinischer Innovation während der Einzelfallbeobachtung und Reflexion gelegt. Klinische Studien sollen dann die formulierten Hypothesen bestätigen oder widerlegen. Die Verantwortung des Klinikers ist, diese Fragen und Hypothesen zu formulieren und sie mit den forschenden, methodologisch kompetenten Kollegen an den akademischen Zentren zu diskutieren. Vielleicht lohnt es sich dann, das eine oder andere Forschungsprotokoll zu schreiben und eine klinische Studie in Kollaboration mit einem Forschungszentrum durchzuführen. Die patientenbetreuenden integrativen Spitäler, Einheiten und Abteilungen sind vorhanden - auch die Institute, Professuren und Lehrstühle mit Forschungsauftrag. Vernetzungen bestehen, können aber klar noch ausgebaut werden. Gute Forschung bedeutet auch für die komplementäre und integrative Medizin neue Erkenntnis und Weiterentwicklung, nicht zuletzt auch gesellschaftliche Akzeptanz und Implementierung. Hierfür ist jeder, der auf dem Feld der integrativen Medizin tätig ist, verantwortlich.

\section{KARGER}

(C) 2016 S. Karger GmbH, Freiburg
Dr. med. Marc Schlaeppi

Zentrum für Integrative Medizin

Kantonsspital St. Gallen

Greithstrasse 20 / Haus 33, 9007 St. Gallen, Schweiz

marc.schlaeppi@kssg.ch 